\title{
Noise suppression system of OCDMA with spectral/spatial 2D hybrid code
}

\author{
Rima, Matem ${ }^{1}$, S. A. Aljunid ${ }^{1}, M . N$ Junita $^{1}$, C. B.M Rashidi $^{1}$, Israa Shihab Aqrab $^{1}$ \\ ${ }^{1}$ Advanced Communication Engineering Centre of Excellence \\ School of Computer and Communication Engineering (ACE CoE -SCCE), \\ Universiti Malaysia Perlis (UniMAP),Perlis, Malaysia,
}

\begin{abstract}
In this paper, we propose a novel 2D spectral/spatial hybrid code based on 1D ZCC and 1D MD where the both present a zero cross correlation property analyzed and the influence of the noise of optical as Phase Induced Intensity Noise (PIIN), shot and thermal noise. This new code is shown effectively to mitigate the PIIN and suppresses MAI. Using 2D ZCC/MD code the performance of the system can be improved in term of as well as to support more simultaneous users compared of the 2D FCC/MDW and 2D DPDC codes.
\end{abstract}

\section{Introduction}

OCDMA has attracted huge research interest according to its advantages features like huge and effective the maximum amount of utilization, higher secure, with high of access of network and dynamic bandwidth assignment. The focus on the development of OCDMA systems is to improve the cardinality, mitigate PIIN (Phase Induced Intensity Noise), as well as suppress MAI (Multiplied Access Inference). Many schemes has been proposed in accordance in the OCDMA system detection such as, frequency hopping [1,2], the time spreading [3-4], spectral amplitude coding (SAC) [5-6] and code of spatial [7]. To have a good OCDMA detection of a code sequence should have the minimum cross-correlation and maximum auto-correlation properties for maintenance.

Several approaches of OCDMA which have been proposed to overcome Phase Induced Intensity Noise [PIIN] reduction and improvement of system of performance [8-9] particularly, in Spectral Amplitude Coding (SAC) OCDMA. For instance, one dimensional (1D) proposed set of code sequences as in [10-11]. The performance is enhanced but the main drawback of the one dimensional code still where the code length become too long as simultaneous users' number increases. To overcome the drawbacks of 1D code, various schemes of 2D (time/spectral, spectral/spatial, etc.) codes have been proposed [12-13]. In comparison with one dimensional sequence for code (1D), the system performance has greatly been improved as well as accommodating more users number within two dimensional codes for (2D) [14].
It became as one of causes to augment enormous research on two dimensional of sequences for (2D) code as it has been proposed in [12][15], to enhance the system of performance and to minimize the Phase Induced Intensity Noise [PIIN]. In the present paper, we do analyze new 2D hybrid code OCDMA based on 1D ZCC code and 1D MD code to examine the effect of Phase Induced Intensity Noise [PIIN] in the system performance.

\section{2D Hybrid ZCC/MD Code}

The 2D-MD code can be constructed based on the combination of 1D ZCC code [15] and 1D MD code [16].Let $X\left\{x_{0}, x_{1}, x_{2}, \ldots, x_{M-1}\right\}, Y\left\{y_{0}, y_{1}, y_{2}, \ldots, y_{N-1}\right\}$ with code weight $k_{1}, k_{2}$ represent 1D-ZCC code and 1DMD code respectively. Noted that $w_{1}, w_{2}$ are the code size of $1 \mathrm{D}-\mathrm{ZCC}$ code and 1D-MD code respectively, so the code length of $X, Y$ are $M=k_{1} w_{1}, N=k_{2} w_{2}$. The code size of 2D-ZCC/MD is $W=w_{1} w_{2}$. Table 1 exhibits some example for sequences of $2 \mathrm{D}$ hybrid $\mathrm{ZCC} / \mathrm{MD}$ codes as follows $k_{1}=3, k_{2}=2$ where $2 \mathrm{D}$ hybrid ZCC/MD codes can be produced by $g=\{1,2,3, \ldots, M\}$ and $h=\{1,2,3, \ldots ., N\} . Y_{g}$ stands for spreading patterns of spectral while $X_{h}$ is the patterns for space.

$$
Z c c=\left[\begin{array}{l}
101000100000 \\
010010001000 \\
000000010101
\end{array}\right] \quad M D=\left[\begin{array}{l}
100001 \\
010010 \\
001100
\end{array}\right]
$$

\footnotetext{
Corresponding author: rymann90@ hotmail.com
} 
Table 1. 2D Hybrid ZCC/MD Code for $k_{1}=3$ and $k_{2}=2$ Sequences

\begin{tabular}{|c|c|c|c|}
\hline$A_{g, i}$ & [101000100000] & [010010001000] & [000000010101] \\
\hline [1] & ] 101000100000 & ¡010010001000 & [000000010101] \\
\hline $0 \mid$ & 000000000000 & 000000000000 & 000000000000 \\
\hline 0 & 000000000000 & 000000000000 & 000000000000 \\
\hline 0 & 000000000000 & 000000000000 & 000000000000 \\
\hline 0 & 000000000000 & 000000000000 & 000000000000 \\
\hline$\lfloor$ ᄂ1 & 101000100000 & لـ & ـ \\
\hline
\end{tabular}

2D ZCC/MD code cross-correlation is derived from 4 matrices of characteristic $(d), d \in(0,1,2,3)$ has been produced in [8] to achieve the same assumption for the cross correlation property as $A(d)$ for 2D Hybrid ZCC/MD codes can be described as follows :

$$
\begin{aligned}
& A^{0}=Y^{T} X \\
& A^{1}=Y^{T} \bar{X} \\
& A^{2}=\overline{Y^{T} X} \\
& A^{3}=\overline{Y^{T} X}
\end{aligned}
$$

Whereas parameters $\bar{X}$ as well as $\bar{Y}$ are representing the whole enchantment of $X \& Y$ appropriately.

The cross correlation of hybrid 2D ZCC/MD code $A^{(d)}$ and $A_{g, h}$ is shown as in the table below:-

$\left.R^{(d)}(g, h)=\right) \sum_{M=1}^{M-1} \sum_{N=1}^{N-1} a_{i j}^{(d)} a_{(i+g)(j+h)}$

Whereas $a_{i j}^{(d)}$ is the $(i, j)_{t h}$ of $A^{(d)}$ and $a_{(i+g)(j+h)}$ is the $(i, j)_{t h}$ of $A_{g, h}$. Table 2 represents the cross correlation is within two different codes $A^{(d)}$ and $A_{g, h}$ for 2D Hybrid ZCC/MD code obtained the equation above.

Table 2. The correlation of $2 \mathrm{D}$ code for $\mathrm{ZCC} / \mathrm{MD}$

\begin{tabular}{|c|c|c|c|c|}
\hline$A_{g, h}$ & $R^{(0)}$ & $R^{(1)}$ & $R^{(2)}$ & $R^{(3)}$ \\
\hline$g=0, h=0$ & $k_{1} k_{2}$ & 0 & 0 & 0 \\
\hline$g=0, h \neq 0$ & 0 & $k_{1} k_{2}$ & 0 & 0 \\
\hline$g \neq 0, h=0$ & 0 & 0 & $k_{1} k_{2}$ & 0 \\
\hline$g \neq 0, h \neq 0$ & 0 & 0 & 0 & $k_{1} k_{2}$ \\
\hline
\end{tabular}

The cross correlation of $A_{0,0}^{0}$ and $A_{g, h}$ can be expressed as follows:

$$
\begin{aligned}
R^{(0)}(g, h) & =\sum_{i=1}^{M-1} \sum_{j=1}^{N-1} a_{i, j}^{(0)} a_{i, j}(g, h) \\
& =\left\{\begin{array}{lr}
k_{1} k_{2} & \text { for } g=0, h=0 \\
0 & \text { otherwise }
\end{array}\right.
\end{aligned}
$$

PIIN (Phase Induced Intensity Noise), Shot Noise and Thermal Noise are considered as three types of noise which one considered in analysis of performance.
BER is basically calculated on Gaussian approximation. The photodiode is employed to detect thermal lights and to obtain the photocurrent as in $[18,19]$ :

$$
\begin{aligned}
& \left\langle i_{\text {noise }}^{2}\right\rangle=\left\langle i_{\text {PIIN }}^{2}\right\rangle+\left\langle i_{\text {shot }}^{2}\right\rangle+\left\langle i_{\text {thermal }}^{2}\right\rangle \\
& =B I^{2} \tau+2 e B I
\end{aligned}
$$

Whereas $I$ stands for the average photocurrent, $e$ is an abbreviation for the electron charge, $B$ means the electrical bandwidth, $K_{b}$ is constant of Boltzmann, $T_{n}$ is the unlimited temperature, $R_{L}$ is the load resistance and $\tau$ is the time for coherence of the light which expressed as [9]:

$$
\tau=\frac{\int_{0}^{\infty} S(v)^{2} d v}{\left[\int_{0}^{\infty} S(v)\right]^{2}}
$$

Where $S(v)$ indicates the density for single sideband power spectral. Since the system of 2D ZCC/MD code has the zero cross property of correlation, there is no overlapping in a spectra of divergent users, thus the effect of MAI have been ignored. Accordingly, by applying the identical method as in [10] where each user probability equally sending bit 1 is equal. We will obtain the output currents of PD at receiver $(0,0)$ is depend on the cross correlation between $A_{0,0}^{(0)}$ and $A_{g, h}$ as follows:

$$
\begin{aligned}
I & =R \int_{0}^{\infty} G(v) d v \\
& =\int_{0}^{\infty} \frac{P_{s r}}{k_{2} \Delta v} \sum_{1}^{w} d(w) R^{0}(i, j) U(v, i) d v \\
& =\frac{P_{s r} \Delta v}{k_{2} \Delta v M}\left[k_{1} k_{2}+\sum_{1}^{w} d(w) R^{0}(i, j)\right] \\
& =\frac{R P_{s r} k_{1}}{M}
\end{aligned}
$$

So the equations of PIIN, shot noise and thermal noise could be written as follow as:

$$
\begin{aligned}
& \left\langle i_{P I I N}^{2}\right\rangle=B \frac{R^{2} P_{S r}^{2} w_{2} k_{1}}{\Delta v W} \\
& \left\langle i_{\text {shot }}^{2}\right\rangle=2 e B \frac{R P_{s r} w_{2}}{W} \\
& \left\langle i_{\text {thermal }}^{2}\right\rangle=\frac{4 K_{b} T_{n} B}{R_{L}}
\end{aligned}
$$

So:

$$
\left\langle i_{\text {noise }}^{2}\right\rangle=\frac{R^{2} P_{s r}^{2} w_{2} k_{1}}{\Delta v W}+2 e B \frac{R P_{s r} w_{2}}{W}+\frac{4 K_{b} T_{n} B}{R_{L}}
$$

where $R$ is the being responsive of the given photodiode given by $R=\eta e / h v_{0}, \eta$ is the efficient. quantum of the photo-diode, and $h$ is the constant of Plank . $P_{s r}$ is the effective source power at the receiver, $k_{1}$ and $k_{2}$ are the weights of code for spectral and spatial accordingly, $W$ represents the active users number, $N$ and $M$ are the code lengths for spatial and spectral code sequences as well . 
Note: that the sending probability bit " 1 " at different time for every user is being $1 / 2$ so:

$\left\langle i_{\text {noise }}^{2}\right\rangle=\frac{R^{2} P_{S r}^{2} w_{2} k_{1}}{2 \Delta v W}+\frac{e B R P_{s r} w_{2}}{W}+\frac{4 K_{b} T_{n} B}{R_{L}}$

At the receiver, the SNR can be obtained as:

$S N R=\frac{I^{2}}{\left\langle i_{\text {noise }}^{2}\right\rangle}=\frac{\left[\frac{R P_{S r} w_{2}}{W}\right]^{2}}{\frac{R^{2} P_{S r}^{2} w_{2} k_{1}}{2 \Delta v W}+\frac{e B R P_{S r} w_{2}}{W}+\frac{4 K_{b} T_{n} B}{R_{L}}}$

The BER can then be calculated from SNR as follows [2]:

$$
B E R=\frac{\operatorname{erfc}(\sqrt{S N R / 8})}{2}
$$

Where

$$
\operatorname{erfc}=\frac{2}{\sqrt{\pi}} \int_{0}^{\infty} \exp \left(-y^{2}\right) d y
$$

Table 3. Used parameters in analytical calculations.

\begin{tabular}{|l|c|}
\hline $\begin{array}{l}\text { PD quantum } \\
\text { efficiency }\end{array}$ & $R=0.75$ \\
\hline $\begin{array}{l}\text { Spectral width of } \\
\text { broadband light } \\
\text { source }\end{array}$ & $\Delta \lambda=30 \mathrm{~nm}(\Delta \lambda=3.75 \mathrm{THZ})$ \\
\hline $\begin{array}{l}\text { Operating } \\
\text { wavelength }\end{array}$ & $\lambda_{0}=1.55 \mu \mathrm{m}$ \\
\hline $\begin{array}{l}\text { Electrical } \\
\text { bandwidth }\end{array}$ & $R_{b}=622 \mathrm{Mbps}$ \\
\hline $\begin{array}{l}\text { Data transmission } \\
\text { rate }\end{array}$ & $T_{n}=300 \mathrm{~K}$ \\
\hline $\begin{array}{l}\text { Receiver noise } \\
\text { temperature }\end{array}$ & $R_{L}=1030 \Omega$ \\
\hline $\begin{array}{l}\text { Receiver load } \\
\text { resistor }\end{array}$ & $K_{b}=1.38 \times 10^{-23} \mathrm{~W} / \mathrm{K} / \mathrm{Hz}$ \\
\hline $\begin{array}{l}\text { Boltzmann's } \\
\text { constant }\end{array}$ & $\mathrm{C}=3 \times 10^{8} \mathrm{~m} / \mathrm{s}$ \\
\hline Electron charge & \\
\hline Light velocity & \\
\hline
\end{tabular}

\section{Result and Discussions}

Table 3 represents the analysis of the newly proposed parameters system performance for 2-D ZCC/MD hybrid OCDMA. The results are numerically shown in the following figures.

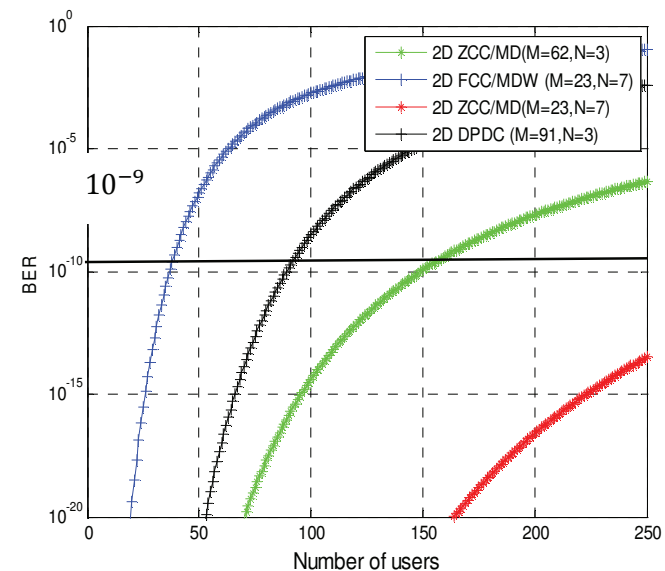

Fig.1. Simultaneous users number which includes only the PIIN noise versus BER

Fig.1 indicates the BER variation versus simultaneous users number for 2D ZCC/MD ( $M=63$, $\mathrm{N}=3)$ compared to 2D FCC/MDW (M=23, N=7) and 2D DPDC ( $M=91, N=3)$ taking into account effective power $\mathrm{Psr}=-10 \mathrm{dBm}$ and data rate $\mathrm{Rb}=2.5 \mathrm{Gbps}$. By neglect shot noise and thermal noise, and considering only PIIN, the result clearly indicates that $2 \mathrm{D}$ ZCC/MD code represents the best performance, where for the BER equal to $10^{-9}$ the proposed code can be support more than 154 of simultaneous users while 2D DPDC can support 98 users and 2D FCC/MDW 43 user. The figure indicates also that the variation of code length show that for $2 \mathrm{D} \mathrm{ZCC}$ $(\mathrm{M}=63, \mathrm{~N}=3)$ can accommodate 154 users where for $2 \mathrm{D}$ ZCC (M=23, N=7) can accommodate until 400 users, so the variation of code length has an effect to the system performance where the proposed code represents always the best cardinality comparing to other codes. The ideal BER is approximately considered beneath $10^{-9}$ error floor with high cardinality. The increase of number of users causes deterioration of the system performance. PIIN noise represents the main effect on the system performance because of increase of code sequences the through higher interference [17] .

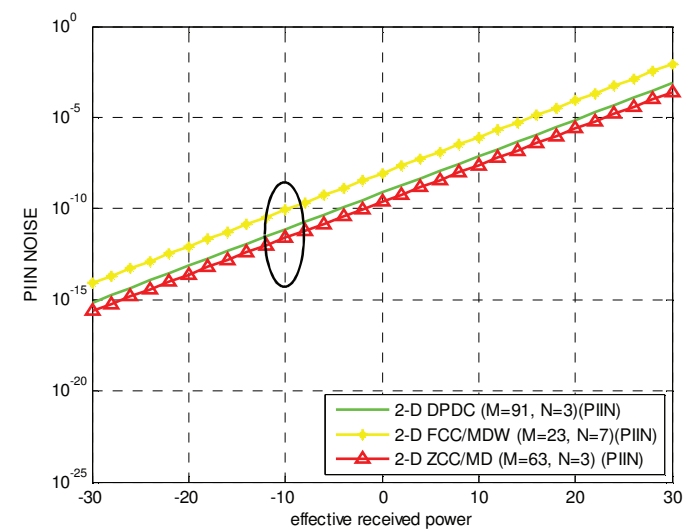

Fig.2. PIIN noise versus effective power when number of user is 80 and data rate s $622 \mathrm{Mbps}$. 
Fig. 2 represents dominant phase-induced intensity noise (PIIN) versus effective power that spreads from $-30 \mathrm{dBm}$ to $30 \mathrm{dBm}$ at $622 \mathrm{Mbps}$ for $2 \mathrm{D}$ ZCC/MD ( $M=63, N=3), 2 \mathrm{D}$ FCC/MDW $(\mathrm{M}=23, \mathrm{~N}=7)$ and 2D DPDC $(\mathrm{M}=91, \mathrm{~N}=3)$. It can be obviously shown the effective increase of the power which leads to increase of noise (PIIN). Comparing 2D ZCC/MD $(\mathrm{M}=63, \mathrm{~N}=3), 2 \mathrm{D}$ FCC/MDW $(\mathrm{M}=23, \mathrm{~N}=7)$ and 2D DPDC $(\mathrm{M}=91, \mathrm{~N}=3)$ codes, PIIN is optimally suppressed by $2 \mathrm{D} \mathrm{ZCC} / \mathrm{MD}$ code. From the figure, below $-10 \mathrm{dBm}$ PIIN can be maximally mitigated. The betterment of OCDMA system performance could be obtained when Psr has low value as possible [17]. The 2D ZCC/MD $(\mathrm{M}=63, \mathrm{~N}=3), 2 \mathrm{D}$ FCC/MDW $(\mathrm{M}=23, \mathrm{~N}=7)$ reaches optimum PIIN suppression is at $-10 \mathrm{dBm}$.

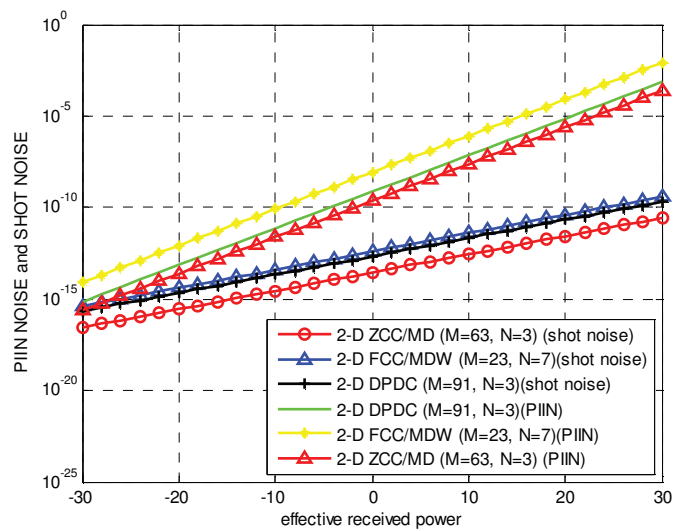

Fig. 3. Phase-Induced Intensity Noise and shot noise versus Psr of effective power within identical codes.

Fig. 3 represents the link between the PhaseInduced Intensity Noise [PIIN] and shot noise versus the source of effective power (Psr). The simultaneous users number is set at 250 and Psr value is various between $30 \mathrm{dBm}$ and $30 \mathrm{dBm}$ range for $2 \mathrm{D} \mathrm{ZCC} / \mathrm{MD}(\mathrm{M}=63$, $\mathrm{N}=3), 2 \mathrm{D}$ FCC/MDW $(\mathrm{M}=23, \mathrm{~N}=7)$ and 2D DPDC $(\mathrm{M}=91, \mathrm{~N}=3)$. From the figure it can be observed that the increase of the effective power Psr produces an increase of PIIN and Shot noise where it attaints the higher values for all codes. For Psr above $-10 \mathrm{dBm}$ Ipiin is dominant and Ishot become negligible. For Psr $=-10 \mathrm{dBm}$, the BER float below $10^{-9}$, Ipiin and Ishot equal to $7.31 \times 10^{-12}, 2.77 \times 10^{-15}$ respectively for $2 \mathrm{D} \mathrm{ZCC} / \mathrm{MD}$ code. $2 \mathrm{D}$ ZCC/MD is better than 2D FCC/MDW and 2D DPDC.

\section{Conclusions}

In the present paper, we study a current/advanced code of $2 \mathrm{D}$ hybrid ZCC/MD in term of noise suppression. We analyze the effect of phase-induced intensity [PIIN] and shot noise on the newly developed of 2D hybrid ZCC/MD OCDMA systems compared to 2D hybrid FCC/MDW and 2D DPDC where 2D hybrid ZCC/MD represents a better performance with effective power fixed at $-10 \mathrm{dBm}$ and it can provide a higher users number when BER equal to $10^{-9}$. PIIN is the main factor in $2 \mathrm{D} \mathrm{ZCC} / \mathrm{MD}$ and others performance degradation. 2D ZCC/MD represents a better performance and effectively mitigates the PIIN.

\section{References}

1. H. Fathallah, Rusch, L. A., \& S. LaRochelle, J. Lightwave Tech., 17(3), p 397, (1999).

2. E. Inaty, Shalaby, H. M., \& Fortier, P., IEEE Trans. Comm., 53 (2), p 323-334, (2005).

3. F. R. Chugh, J.A. Salehi, \& V. K. Wei., IEEE Trans. Inf. Thery, 35(3), (1989).

4. C.S. Weng, \& J. Wu., J. Lightwave Tech., 19(2), p.186. (2001).

5. E.D. Smith, R. J. Blaikie, \& D. P. Taylor, IEEE Trans. Comm., 46(9),p 1176-1185. (1998).

6. A.T. Hussein, S. A. Aljunid, S. Junid, H. Adnan, \& R. Badlishah, Aust. J. Basic \& Appl. Sci., 6 (3), p112 (2012).

7. K.I. Kitayama, IEEE J. on Sel. Areas in Comm., 12(4), p762-772. (1994).

8. M. S. Anuar, S. A. Aljunid, N. M. Saad \& S. M. Hamzah, Optics Comm., 282(14), p.2659-2664. (2009).

9. Z. Wei, H. M. H. Shalaby, \& H., Ghafouri-Shiraz, J. Lightwave Tech., 19(9), p 1274-1281. (2001).

10. S. A. Aljunid, M. D. A. Samad, M. Othman, M. H. Hisham, A. H. Kasiman, \& M. K Abdullah, IEEE 7th Malaysia International Conference on Communication, 2005, 1, pp. 5 (2005).

11. J. F. Huang, \& Y.T Chang., Communication Networks and Services Research Conference, 2006. CNSR 2006. Proceedings of the 4th Annual, pp. 8 (2006).

12. S. Wan, X. He \& J .Sun, Optics Comm., 282(24), p4776-4778. (2009).

13. B. C. Yeh, C. H.Lin., C.L. Yang, \& J. Wu., J. Lightwave Tech., 27(13), 2420-2432. (2009).

14. N. D. Keraf, S. A. Aljunid, A. R. Arief, M. N. Nurol, M. S. Anuar, C. B. M. Rashidi, \&, P.Ehkan, 2014 IEEE 5th International Conference on Photonics, pp. 166-168. (2014).

15. M.S. Anuar, S.A. Aljunid, N.M. Saad. IJCSNS Int. J. Comp. Sci. and Netw. Sec., 6 (12), pp. 180184. (2006).

16. R.A. Kadhim, H. A. Fadhil, S. A. Aljunid \& M. S Razalli. Optics Comm., 329, pp. 28-33. (2014).

17. A. Arief, S. Aljunid, M. Anuar, M.N. Junita, R. Ahmad, and F. Ghani, IEEE 3rd International Conference on Photonics. pp. 275-279. (2012).

18. B.C. Yeh, C.H. Lin \& J. Wu. J. Opt. Comm. Netw. 2(9), pp. 653-661. (2010).

19. Z. Wei and H. Ghafouri-Shiraz, J. Lightwave Tech., 20, pp. 1284-1289. (2002). 